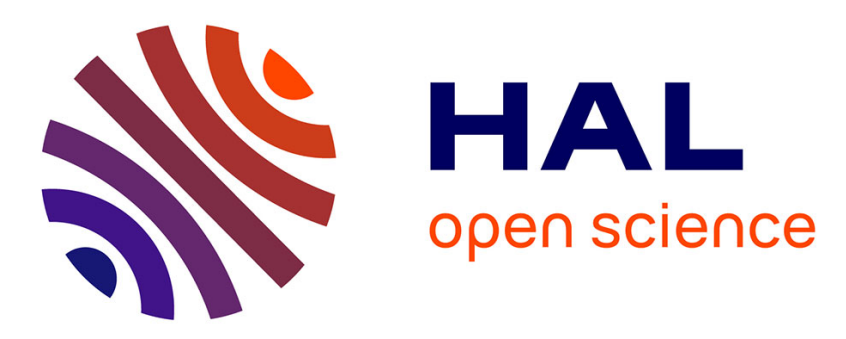

\title{
Modelling and control for position-controlled modular robot manipulators
}

\author{
Zilong Shao, Gang Zheng, Denis Efimov, Wilfrid Perruquetti
}

\section{To cite this version:}

Zilong Shao, Gang Zheng, Denis Efimov, Wilfrid Perruquetti. Modelling and control for positioncontrolled modular robot manipulators. Proc. IEEE/RSJ Int. Conf. Intelligent Robots and Systems (IROS), Sep 2015, Hamburg, Germany. hal-01185120

\section{HAL Id: hal-01185120 \\ https://inria.hal.science/hal-01185120}

Submitted on 5 Oct 2015

HAL is a multi-disciplinary open access archive for the deposit and dissemination of scientific research documents, whether they are published or not. The documents may come from teaching and research institutions in France or abroad, or from public or private research centers.
L'archive ouverte pluridisciplinaire HAL, est destinée au dépôt et à la diffusion de documents scientifiques de niveau recherche, publiés ou non, émanant des établissements d'enseignement et de recherche français ou étrangers, des laboratoires publics ou privés. 


\title{
Modelling and control for position-controlled modular robot manipulators
}

\author{
Zilong Shao ${ }^{1,2}$ Gang Zheng ${ }^{1,2}$ Denis Efimov ${ }^{1,2}$ Wilfrid Perruquetti ${ }^{1,2}$
}

\begin{abstract}
Modular Robot Manipulators are userconfigurable manipulators which provide rapid design and inexpensive implementation. To be easy-use, smart actuators embedded with position input and position feedback controller are adopted, these local controllers render the manipulators position controlled, but also result in limited performance and precision. This paper targets the case that the built-in controller does not provide desirable precision for set-point regulation. Firstly a joint-level model is established, of which the nominal model can be identified with derivative observer based on the position feedback, then an auxiliary adaptive controller coping with parametric uncertainty is proposed which leads to an error close to zero, a switching control strategy is introduced considering the actuator saturation. The proposed control method is implemented on a 5-DOF modular manipulator, with comparison to classic integral controller.
\end{abstract}

\section{INTRODUCTION}

Modular robots can be assembled by the users with various modules, these modules include physical links, actuators, sensors, end-effectors, computing systems and other components. Apart from its relative low price and light weight, modular robot manipulators are reconfigurable to various tasks, easy to repair since defective module can be easily replaced.

To be easy-use for even non-professionals, modular robots adopt usually smart actuators, which provides built-in controllers with position feedback and position input, these jointlevel controllers render the manipulators position controlled, but also result in limited performance and precision [3], since the highly coupled dynamic effects between joints are ignored [2].

Since torque controlled robots are preferred to achieve high performance, works have been done to provide position controlled robots with torque control capability. Approach to use torque sensors such as strain gauge [6] or optical torque sensor [7] techniques is proposed in [4], this joint torque sensing method is adopted in [5]. However, since strain gauges are sensitive to ambient temperature variations, varying offset are often generated. For optical torque sensors, artificial flexibility inside a joint usually needs to be performed to convert the joint torque to joint deformation, while introducing joint flexibility may cause undesired effects such as vibration. In [8], a torque-position transformer for position controlled robots is introduced, with which desired joint

\footnotetext{
${ }^{1}$ CRIStAL (UMR CNRS 9189) Ecole Centrale de Lille,Cité Scientifique, 59651, Villeneuve d'Ascq, France.

${ }^{2}$ Non-A team, INRIA -Lille Nord Europe, 40 avenue Halley, 59650 Villeneuve d'Ascq, France. (zilong.shao, gang.zheng, denis.efimov, wilfrid.perruquetti) @inria.fr
}

torque is converted into instantaneous increments of joint position inputs, however, this transformer is based on the total knowledge of the built-in controller, which is usually unknown to individual users.

Besides the lack of torque-control ability, some other operational constraints also limit the performance for some modular robots. Firstly, instead of PD controller, which is the most used closed-loop control of a servo system [9], simple $\mathrm{P}$ controller is adopted, due to the fact that cheap actuators generally do not provide reliable velocity measurement, thus under certain load, the steady state error cannot be negligible. Secondly, those cheap and simple actuators get hot easily under high output torque or high velocity, high temperature leads to system shut-down or burnout of the circuit, thus output torque and moving velocity are saturated. Thirdly, due to limited stiff materials, one advantage or disadvantage lies in the flexibility, which is more secure for human-machine interaction, but leads also to vibration, for applications where vibration is not desired, the moving velocity needs be further limited.

As a following work of [10], this paper aims at improving the precision of set-point control for manipulators under embedded $\mathrm{P}$ controller, with presence of torque saturation and velocity limitation, without using any supplementary sensors. We also constraint ourselves as individual users who have no knowledge of the embedded controller parameter o the physical joint model.

The paper is organized as follows: The problem statement is clarified in section II. In section III, local modelling of the controlled system is developed and derivative observers are introduced. The auxiliary controller design and control strategy are presented in Section IV. Section V is devoted to the experimental implementation and results. Finally, some concluding remarks are given in Section VI.

\section{Problem StATEMEnT}

We are facing a $n$-DOF modular robot manipulator driven by $n$ actuators, each actuator is embedded with its own position controller which presents undesired steady state error under load.

Fig. 1 shows a block diagram of a position feedback loop for independent joint-level embedded control. $x_{d}, x, e$ and $\tau$ are respectively the desired joint angular position, joint angular position, position error and joint torque. Specifically, the built-in controller $D$ is a proportional controller with saturation as illustrated in Fig. 2.

The following assumptions are made: 


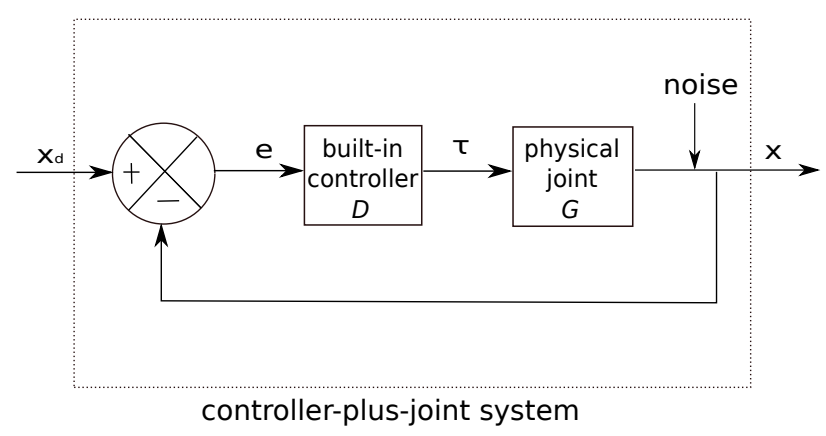

Fig. 1. Block diagram of the embedded control

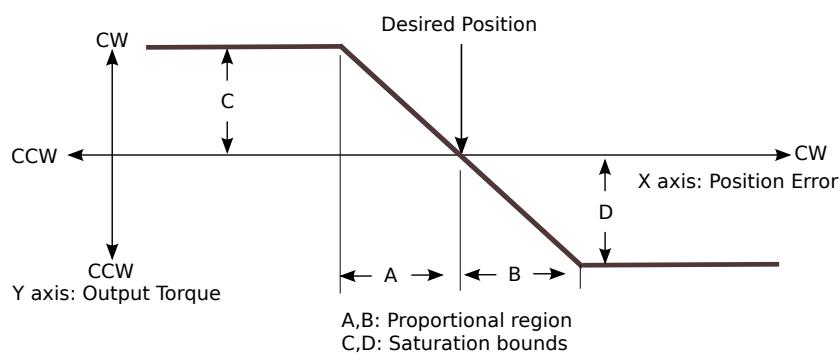

Fig. 2. D: Proportional built-in controller with saturation

Assumption 1. The proportional region of $D$ is known (e.g., Dynamixel AX-12 series).

Assumption 2. Both the joints and the links are rigid, thus flexibility is not considered.

Assumption 3. Time delay is negligible.

Assumption 4. Precise trajectory tracking is not considered, since the tracking performance is affected by nonlinear effects (e.g. actuator saturation) for low-cost manipulators.

Several constraints are also imposed as bellow:

Constraint 1 . The saturation bounds and the proportional gain of the built-in controller $D$ are unknown.

Constraint 2. The joint model $G$ is unknown.

Constraint 3. Only joint angular position feedback is available and is corrupted with noise.

To improve the control precision for a set-point regulation, our idea is to design the desired position input $x_{d}$, by adding an auxiliary controller $E$ as illustrated in Fig. 3

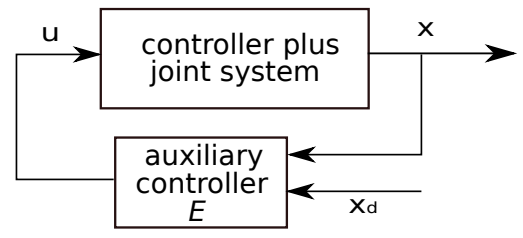

Fig. 3. Block diagram with addition of an auxiliary controller

As for the design of the auxiliary controller, an integral controller with a constant gain $K_{i}$ described as

$$
u=K_{i} \int\left(x_{d}-x\right),
$$

may be a simple choice, since it does not need a precise model of the system or any derivative of the measured position, however, an integral term with a constant gain may not be suitable for a nonlinear and time-varying actuator system, from performance viewpoint. To seek a better performance, a model-based controller could be a better choice, so we try to formulate a decoupled individual model for each joint.

\section{ModelLing AND IDENTIFICATION}

\section{A. Modelling}

Generally, the Lagrange formulated dynamics of an $n$-DOF robot arm can be denoted as:

$$
M(q) \ddot{q}+V_{m}(q, \dot{q}) \dot{q}+F(q, \dot{q})+G(q)=\Gamma,
$$

where $q$ is the joint variable $n$-vector and $\Gamma$ is the $n$-vector of the generalized forces or torques. $M(q)$ is the inertia matrix, $V_{m}(q, \dot{q}) \dot{q}$ is the Coriolis/centripetal $n$-vector, $F(q, \dot{q})$ is the friction vector, and $G(q)$ is the gravity vector. One of the properties of (2) is that $M(q), V_{m}(q, \dot{q}), F(q, \dot{q}), G(q), \Gamma$ are bounded [11].

The model (2) is highly coupled, to get a decoupled individual model for each joint, we extract:

$$
m(t) \ddot{x}+n(t) \dot{x}+c(t) x+e(t)=\tau(t),
$$

where $\tau(t)$ and $x$ represent respectively the torque and joint position for the corresponding link, $m(t), n(t), c(t)$ are the parameters for linear terms, $e(t)$ consists of the modelling error and disturbance. Because of the boundedness of the terms in (2), it can be concluded that $\tau(t), m(t), n(t), c(t)$ and $e(t)$ are bounded.

The proportional controller $D$ can be expressed as

$$
\tau(t)=k_{p}\left(x_{d}-x\right)
$$

with $k_{p}$ the bounded proportional gain and $x_{d}$ the desired position. Now we have

$$
m(t) \ddot{x}+n(t) \dot{x}+c(t) x+e(t)=k_{p}\left(x_{d}-x\right),
$$

which leads to

$$
\ddot{x}=-\frac{c(t)+k_{p}}{m(t)} x-\frac{n(t)}{m(t)} \dot{x}+\frac{k_{p}}{m(t)} x_{d}-\frac{1}{m(t)} e(t) .
$$

Take $x_{d}$ as the input $u$ to the controller-plus-joint system, then (6) becomes

$$
\ddot{x}=-a_{1}(t) x-a_{2}(t) \dot{x}+k(t) u+\lambda(t),
$$

where $a_{1}(t)=\frac{c(t)+k_{p}}{m(t)}, a_{2}(t)=\frac{n(t)}{m(t)}, k(t)=\frac{k_{p}}{m(t)}, \lambda(t)=$ $-\frac{1}{m(t)} e(t)$. The boundedness of the terms in (3) leads to the boundedness of $a_{1}(t), a_{2}(t), k(t)$ and $\lambda(t)$. In what follows, we are going to estimate a linear nominal model of (7) with constant parameters $\bar{a}_{1}, \bar{a}_{2}, \bar{k}$, without the modelling error and disturbance term $\lambda(t)$. 


\section{B. Estimation of the nominal parameters}

Considering a linear nominal model of (7) as follows:

$$
\ddot{x}=-\bar{a}_{1} x-\bar{a}_{2} \dot{x}+\bar{k} u,
$$

where $\bar{a}_{1}, \bar{a}_{2}, \bar{k}$ are constant. (8) can be rewritten in the following matrix form:

$$
\ddot{x}=\left[\begin{array}{lll}
x & \dot{x} & u
\end{array}\right]\left[\begin{array}{lll}
-\bar{a}_{1} & -\bar{a}_{2} & \bar{k}
\end{array}\right]^{T} .
$$

Denoting $A=\left[\begin{array}{c}\ddot{x}\left(t_{1}\right) \\ \ddot{x}\left(t_{2}\right) \\ \vdots \\ \ddot{x}\left(t_{n}\right)\end{array}\right], B=\left[\begin{array}{ccc}x\left(t_{1}\right) & \dot{x}\left(t_{1}\right) & u\left(t_{1}\right) \\ x\left(t_{2}\right) & \dot{x}\left(t_{2}\right) & u\left(t_{2}\right) \\ \vdots & \vdots & \vdots \\ x\left(t_{n}\right) & \dot{x}\left(t_{n}\right) & u\left(t_{n}\right)\end{array}\right]$ and $C=\left[\begin{array}{lll}-\bar{a}_{1} & -\bar{a}_{2} & \bar{k}\end{array}\right]^{T}$, then we can use least square method to estimate those parameters in $C$ :

$$
C=\left(B^{T} B\right)^{-1} B^{T} A
$$

It is worth noting that the above method is based on the knowledge of the velocity $\dot{x}$ and the acceleration $\ddot{x}$, since we have only position feedback $x$, therefore, we need to estimate $\dot{x}$ and $\ddot{x}$ by using high-order observers.

\section{Derivative observers}

Suppose the position feedback is corrupted by a zeromean noise. For an off-line work, to estimate the derivatives, using a certain window of measurements results in smoother results, and the delay caused by the window can be removed by a shifting. Thus the algebraic-based observer (Alien) [16], [17] is suitable for the off-line nominal parameters estimation task, to estimate the first and second derivatives. However, for on-line work, since this delay cannot be removed, homogeneous observer (HOMD) [14], [15] giving finite-time observation is adopted, which serves the controller computation detailed in section IV. To give a formulation of these two observers, let $y(t)=x(t)+w(t)$ be a noisy observation of a signal $x(t)$ with noise $w(t)$.

1) Algebraic-based observer: The basic idea of this approach is to approximate the noisy signal by a suitable polynomial during a small time window. In a practical way, the first-order and second-order derivative estimates can be expressed as (refer to [17] for details):

$$
\begin{gathered}
\hat{\dot{y}}(t)=-\frac{6}{T} \int_{0}^{1}(1-2 \tau) y(t-\tau T) d \tau, \\
\hat{y}(t)=\frac{60}{T^{2}} \int_{0}^{1}\left(6 \tau^{2}-6 \tau+1\right) y(t-\tau T) d \tau,
\end{gathered}
$$

where $y$ is the corrupted signal and $T$ is the window time.
2) Finite-time homogeneous observer: The recursive schemes of the homogeneous observer can be described as follows:

$$
\begin{aligned}
& \dot{z}_{1}=-k_{1}\left\ulcorner z_{1}-y\right\lrcorner^{\alpha}+z_{2} \\
& \vdots \\
& \dot{z}_{i}=-k_{i}\left\ulcorner z_{1}-y\right\lrcorner^{i \alpha-(i-1)}+z_{i+1} \\
& \vdots \\
& \dot{z}_{n-1}=-k_{n-1}\left\ulcorner z_{1}-y\right\lrcorner^{(n-1) \alpha-(n-2)}+z_{n} \\
& \dot{z}_{n}=-k_{n}\left\ulcorner z_{1}-y\right\lrcorner^{n \alpha-(n-1)}
\end{aligned}
$$

where $\ulcorner a\lrcorner^{b}=|a|^{b} \operatorname{sign}(a)$ and $k_{i}$ is the chosen gain. Then $z_{i}$ represents the estimate of the $i$ th order derivative of $y$. According to [14], by choosing $\alpha \in\left(\frac{n-1}{n}, 1\right)$, finite time observation can be obtained.

\section{AUXILIARY CONTROLLER DESIGN AND CONTROL STRATEGY}

\section{A. Auxiliary controller design}

The model of the controller-plus-joint system (7) can be rewritten in the following form:

$$
\ddot{x}=-\bar{a}_{1} x-\bar{a}_{2} \dot{x}+\bar{k}\left(u+\varphi^{T} \theta+d\right),
$$

where $\bar{a}_{1}, \bar{a}_{2}, \bar{k}$ are the nominal parameters of (8), and

$$
\varphi=\left[\begin{array}{lll}
x & \dot{x} & u
\end{array}\right]^{T}, \theta=\left[\begin{array}{ccc}
\frac{\bar{a}_{1}-a_{1}(t)}{\bar{k}} & \frac{\bar{a}_{2}-a_{2}(t)}{\bar{k}} & \frac{k(t)-\bar{k}}{\bar{k}}
\end{array}\right]^{T},
$$

where $\theta$ represents the parameter uncertainties, and $d=$ $\lambda(t) / \bar{k}$ represents time-varying disturbance as introduced in (7). The boundedness of the parameters in (7) leads to the boundedness of $\theta$ and $d$.

Take $x_{d}$ as the trajectory reference, particularly, for a setpoint regulation problem, $\ddot{x_{d}}=\dot{x_{d}}=0$. We suppose $\bar{a}_{1}>0$ and $\bar{a}_{2}>0$, from [18], the control law is given by

$$
u=\bar{k}^{-1} \bar{a}_{1} x_{d}-\varphi^{T} \hat{\theta},
$$

where $\hat{\theta}$ denotes the estimate of $\theta$ and will be derived hereafter.

Remark 1. $u$ is the input, while the right hand side of (15) includes $u$, in discrete system, just need to take the value a step before to derive the current $u$, as a common practice in digital devices. In fact, a form $u=\bar{b}_{1} x-\bar{b}_{2} \dot{x}+\bar{b}_{3} \ddot{x}+\varphi^{T} \theta+d$ instead of (14) with $\varphi=\left[\begin{array}{ccc}x & \dot{x} & \ddot{x}\end{array}\right]^{T}$ can avoid this issue, but results in estimate of $\ddot{x}$, which can hardly be reliable since $x$ is corrupted.

After substituting the control law above into (14), we can form the error system

$$
\ddot{e}=-\bar{a}_{1} e-\bar{a}_{2} \dot{e}+\bar{k}\left(\varphi^{T}(\theta-\hat{\theta})+d\right),
$$

where $e=x-x_{d}$. Note $E=\left[\begin{array}{c}e \\ \dot{e}\end{array}\right]$ and $\tilde{\theta}=\theta-\hat{\theta}$, then we get

$$
\dot{E}=A E+B\left(\varphi^{T} \tilde{\theta}+d\right) \text {, }
$$

with $A=\left[\begin{array}{cc}0 & 1 \\ -\bar{a}_{1} & -\bar{a}_{2}\end{array}\right]$ and $B=\left[\begin{array}{l}0 \\ \bar{k}\end{array}\right]$. 
Now select the positive-definite Lyapunov-like function

$$
V=E^{T} P E+\tilde{\theta}^{T} \tilde{\theta},
$$

where $P$ is a positive-definite constant symmetric matrix. The time derivative of $V$ equals:

$$
\dot{V}=-E^{T} Q E+2\left(E^{T} P B \varphi^{T}+\dot{\tilde{\theta}}^{T}\right) \tilde{\theta}+2 E^{T} P B d,
$$

where $Q$ is positive definite defined by

$$
Q=-\left(P A+A^{T} P\right) \text {. }
$$

By taking

$$
\dot{\tilde{\theta}}=-\left(\varphi B^{T} P E-\kappa \hat{\theta}\right),
$$

where $\kappa>0$, then (19) becomes:

$$
\dot{V}=-E^{T} Q E-\kappa \tilde{\theta}^{T} \tilde{\theta}+\kappa \theta^{T} \theta-\kappa \hat{\theta}^{T} \hat{\theta}+2 E^{T} P B d .
$$

Apply Young's inequality, we have

$$
\begin{gathered}
2 E^{T} P B d=2 E^{T} \frac{Q^{\frac{1}{2}}}{\sqrt{2}} * \sqrt{2} Q^{-\frac{1}{2}} P B d \\
\leq \frac{1}{2} E^{T} Q E+2 d^{T} B^{T} P^{T} Q^{-1} P B d .
\end{gathered}
$$

Note $\lambda_{\min }(Q), \lambda_{\max }(P)$ and $\delta$ respectively the minimal eigenvalue of $Q$, the maximal eigenvalue of $P$ and the maximal eigenvalue of $B^{T} P^{T} Q^{-1} P B$, since $P$ and $Q$ are positive definite, $\lambda_{\min }(Q)>0$ and $\lambda_{\max }(P)>0$, then we have

$$
-E^{T} Q E \leq-\lambda_{\min }(Q)|E|^{2} \leq-\frac{\lambda_{\min }(Q)}{\lambda_{\max }(P)} E^{T} P E,
$$

and

$$
2 d^{T} B^{T} P^{T} Q^{-1} P B d \leq 2 \delta|d|^{2} \leq 2|\delta||d|^{2} .
$$

With (23), (24) and (25), we get

$$
\dot{V} \leq-\frac{\lambda_{\min }(Q)}{2 \lambda_{\max }(P)} E^{T} P E-\kappa \tilde{\theta}^{T} \tilde{\theta}+\kappa \theta^{T} \theta+2|\delta||d|^{2} .
$$

Since $\theta$ and $d$ are bounded, then we can define $\beta=$ $\sup \left(\kappa \theta^{T} \theta+2|\delta||d|^{2}\right)$ and $\alpha=\min \left(\frac{\lambda_{\min }(Q)}{2 \lambda_{\max }(P)}, \kappa\right)$, it is obvious that $\alpha>0$ and $\beta>0$, then we have

$$
\dot{V} \leq-\alpha V+\beta .
$$

As time tends to infinity, $V_{t \rightarrow \infty}$ will be bounded by $\frac{\beta}{\alpha}$, from (18), we derive

$$
\lambda_{\text {min }}(P)\left|E_{t \rightarrow \infty}\right|^{2} \leq E_{t \rightarrow \infty}^{T} P E_{t \rightarrow \infty} \leq V_{t \rightarrow \infty} \leq \frac{\beta}{\alpha},
$$

where $\lambda_{\min }(P)$ is the minimal eigenvalue of $P$. This gives

$$
\left|E_{t \rightarrow \infty}\right| \leq \sqrt{\frac{\beta}{\alpha \lambda_{\min }(P)}} .
$$

So, finally $E_{t \rightarrow \infty}$ will be bounded. Further more, if $\kappa$ and $P$ are well chosen, especially when $\lambda_{\min }(P)$ is large enough, then an error close to zero can be obtained.

Further more, if the control region for $x$ is rather narrow and the moving speed $\dot{x}$ is limited, $a_{1}(t), a_{2}(t)$ and $k(t)$ of (7) can be considered as unknown piece-wise constant, this derives that $\theta$ is also piece-wise constant, i.e., $\dot{\theta}=0$, then we obtain the adaptive update rule for $\hat{\theta}$ as

$$
\dot{\hat{\theta}}=-\dot{\tilde{\theta}}=\varphi B^{T} P E-\kappa \hat{\theta} .
$$

\section{B. Control strategy}

The performance degrades under closed-loop control with saturation, in our case, the output torque is saturated and the velocity is limited, besides, the adaptive update rule (30) imposes a narrow control region, thus a switching control strategy is introduced. For this, let us firstly define a narrow neighbourhood $\Omega$ around the desired position $x_{d}$ :

$$
\Omega=\left\{x:\left|x-x_{d}\right| \leq \omega\right\},
$$

where $\omega$ is a pre-defined distance. Outside of this region, i.e. $x \notin \Omega$, we use only the built-in controller, since trajectory tracking is not considered, the advantage of this phase is the rapidity due to the torque or velocity saturation. Within this region, we activate this auxiliary controller, which generates $u_{2}$ served as the input for the system. The switching controller is described as

$$
u= \begin{cases}x_{d}, & \text { if } x \notin \Omega \\ u_{2}, & \text { if } x \in \Omega .\end{cases}
$$

This witching control strategy can be summarized as Fig. 4

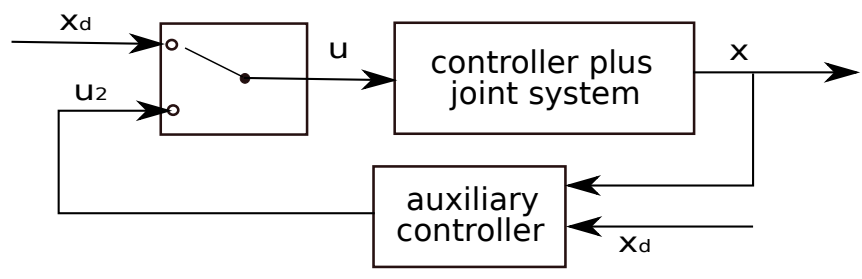

Fig. 4. Switched control strategy

\section{EXPERIMENTAL RESULTS}

\section{A. Test Manipulator Introduction}

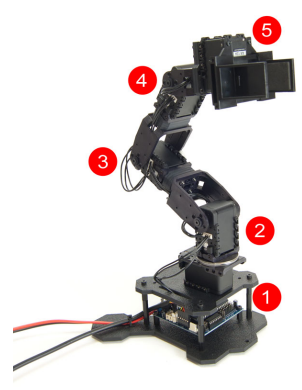

Fig. 5. 5 DOF Manipulator

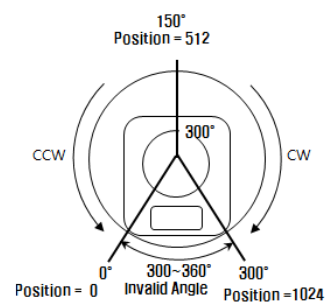

Fig. 6. Angle range
The proposed auxiliary controller has been implemented on a 5-DOF modular robot manipulator, as shown in Fig. 5. Each joint of the robot contains an Dynamixel AX$12 \mathrm{~A}$ actuator embedded with a position controller. Posed 
on a horizontal plane, the shoulder-pan joint (1st joint) gives a rotation about the vertical axis, while the axes of the shoulder-lift joint, the elbow joint and the wrist joint are parallel and horizontal, the last joint is attached to the gripper. In joint mode, the range of the actuator is 0$300^{\circ}$ with the peak position noted as $150^{\circ}$, with a resolution of $300 / 1024=0.29^{\circ}$, as shown in Fig. 6 , we use this position notation in what follows. Among the available feedbacks of the actuators, only position feedback is of interest and reliable [19]. The 5 actuators are connected, by a bus to an Arduino controller board where the computation occurs, the controller board is connected with a FTDI Cable to a PC where the command is sent and the data is collected.

The built-in controller is exactly of the form we assumed in Section II, proportional controller with saturation.

\section{B. Implementation settings}

As the load gravity does not exert torque on the vertical axis of the shoulder-pan actuator, thus the steady state error is not evident, while the gripper needs more a compliant control rather than a precise position control, our auxiliary controller is implemented on the middle 3 actuators. Due to the computational time consumption on the Arduino board and the data updating and transmission, the sampling interval is $15 \mathrm{~ms}$.

For this actuator, the proportional region is very limited with a maximum value of $128 * 0.29=37.5^{\circ}$, besides of the torque saturation, the maximal velocity is limited as well, to protect our auxiliary controller from these saturations, we choose the switching region $\omega=64 * 0.29=18.8^{\circ}$.

\section{Observer performance}

Algebraic-based observer and finite-time homogeneous observer are used respectively for parameter estimation task and controller computation task. The performance of the two observers is shown in Fig. 7.
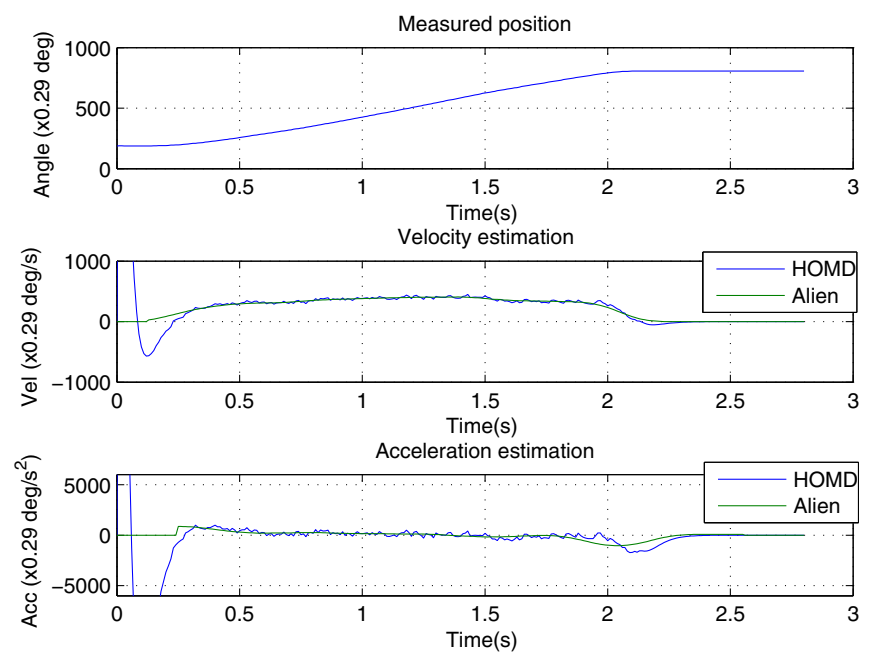

Fig. 7. First-order and second-order derivative estimates
From the feedback position, velocity saturation is clearly observed. As for the first-order derivative estimate, apart from the transient period of the finite-time homogeneous observer, the results are quite close, while for second-order derivative estimate, the result of the finite-time homogeneous observer is quite oscillatory.

\section{Parameter estimation results}

The nominal models are identified with only built-in controller. To avoid saturation, the input signals are designed such that the position error is always within the proportional region. The results for shoulder-lift, elbow and wrist joints are shown respectively in (32), (33) and (34).

$$
\begin{aligned}
\ddot{x} & =-32.52 x-3.07 \dot{x}+53.13 u, \\
\ddot{x} & =-34.86 x-4.42 \dot{x}+34.26 u . \\
\ddot{x} & =-48.53 x-4.30 \dot{x}+47.66 u .
\end{aligned}
$$

\section{E. Control performance}

The comparison of the control performance with only built-in controller, with auxiliary integral controller and with auxiliary adaptive controller under different scenarios is shown in Fig. 8, and in Fig. 9 where an additional load is added to the grasper. The auxiliary controller parameters are determined by trials and errors. The initial position is $\left(148.5^{\circ}, 148.5^{\circ}, 148.5^{\circ}\right)$, respectively for shoulder-lift, elbow and wrist joints, and the goal position is $\left(232^{\circ}, 58^{\circ}\right.$, $232^{\circ}$ ). A more complete experiment video is available at https://youtu.be/0-Yxy4lg7O0.

Under only built-in controller, since the gravity load torque depends on the load gravity and the load configuration, the steady state error for the wrist joint is rather small and is evident for shoulder-lift joint, while for elbow joint it can be reduced for some particular position of the wrist Fig. 8(a). However, when the additional load is added, the error increases for each joint.

With addition of an auxiliary controller, with or without additional load, the errors are attenuated to a close to zero level for both scenarios and both integral controller and adaptive controller.

However, since the integral controller gains are tuned for scenario A without additional load, integral controller manifests good performance for Fig. 8(b), when additional load is added or scenario changes, the performance degrades with more obvious overshoot and prolonged convergence time. While for the proposed adaptive controller, the good performance persists for all operational conditions.

\section{CONCLUSIONS}

The lack of control precision of position controlled modular robot manipulators, under embedded proportional controller has been investigated. Without using supplementary sensors, an adaptive auxiliary controller and a switching control strategy are proposed to respectively cope with parametric uncertainty and actuator saturation. The experimental results showed powerful ability of the proposed auxiliary 


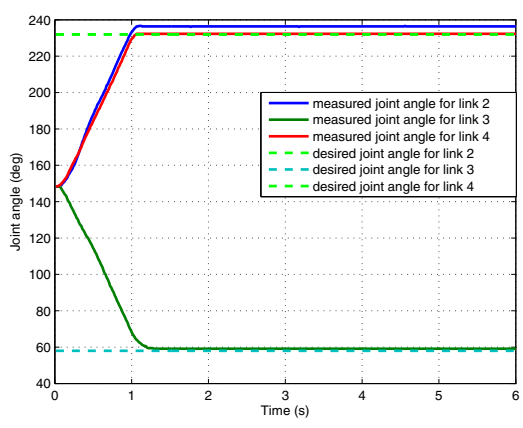

(a) Only built-in controller

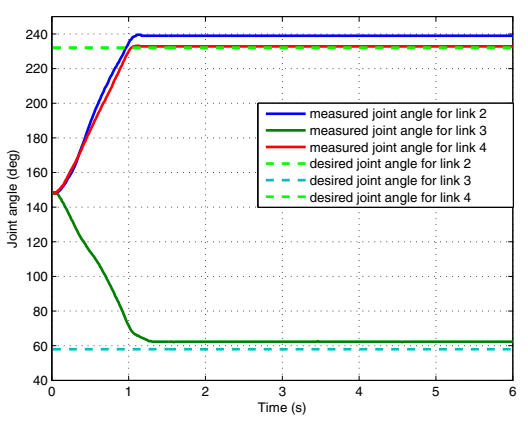

(a) Only built-in controller

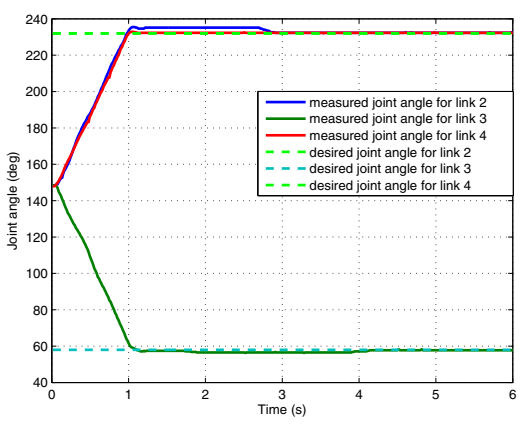

(b) Addition of integral controller

Fig. 8. Without additional load

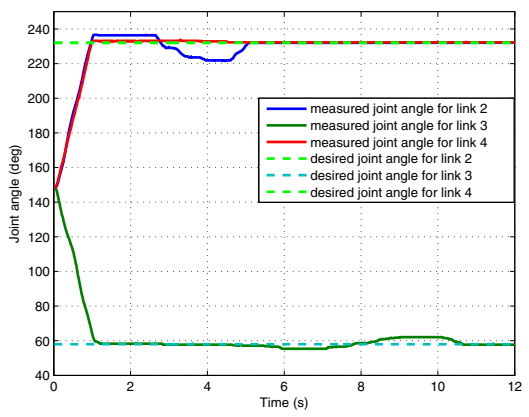

(b) Addition of integral controller

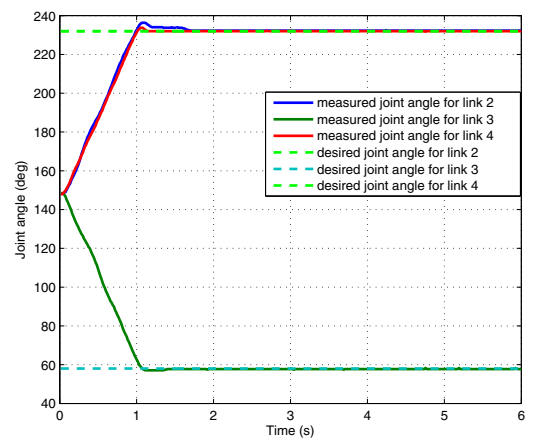

(c) Addition of adaptive controller

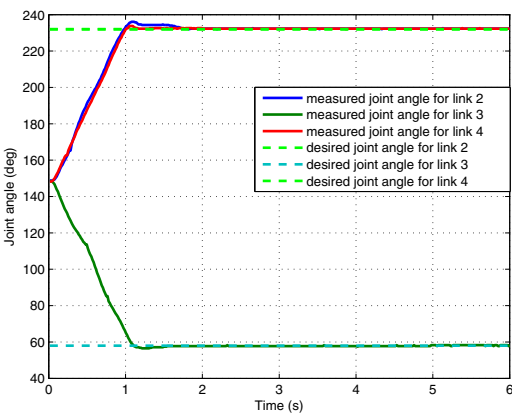

(c) Addition of adaptive controller

Fig. 9. With additional load

controller in error attenuation, and its advantage over classic integral controller with constant gain in quick convergence viewpoint.

\section{REFERENCES}

[1] Yim M, Shen W M, Salemi B, et al. Modular self-reconfigurable robot systems [grand challenges of robotics][J]. Robotics and Automation Magazine, IEEE, 2007, 14(1): 43-52.

[2] Craig J J. Introduction to robotics: mechanics and control[M]. Upper Saddle River: Pearson Prentice Hall, 2005.

[3] Zhu W H, Lamarche T. Modular robot manipulators based on virtual decomposition control[C]//Robotics and Automation, 2007 IEEE International Conference on. IEEE, 2007: 2235-2240.

[4] Hirzinger G, Albu-Schaffer A, Hahnle M, et al. On a new generation of torque controlled light-weight robots[C]//Robotics and Automation, 2001. Proceedings 2001 ICRA. IEEE International Conference on. IEEE, 2001, 4: 3356-3363.

[5] Liu G, Abdul S, Goldenberg A A. Distributed control of modular and reconfigurable robot with torque sensing[J]. Robotica, 2008, 26(01): 75-84.

[6] Hashimoto M, Kiyosawa Y, Paul R P. A torque sensing technique for robots with harmonic drives[J]. Robotics and Automation, IEEE Transactions on, 1993, 9(1): 108-116.

[7] Hirose S, Yoneda K. Development of optical six-axial force sensor and its signal calibration considering nonlinear interference[C]//Robotics and Automation, 1990. Proceedings., 1990 IEEE International Conference on. IEEE, 1990: 46-53.

[8] Khatib O, Thaulad P, Yoshikawa T, et al. Torque-position transformer for task control of position controlled robots[C]/Robotics and Automation, 2008. ICRA 2008. IEEE International Conference on. IEEE, 2008: 1729-1734.

[9] Goldfarb M, Sirithanapipat T. The effect of actuator saturation on the performance of PD-controlled servo systems[J]. Mechatronics, 1999, 9(5): 497-511.
[10] Shao Z, Zheng G, Efimov D, et al. Modelling and control of actuators with built-in position controller[C]//IFAC MICNON 2015. 2015.

[11] Bernstein D S, Michel A N. A chronological bibliography on saturating actuators[J]. International Journal of robust and nonlinear control, 1995, 5(5): 375-380.

[12] Lewis FL, Dawson DM, Abdallah CT. Robot Manipulator Control:Theory and Practice. NewYork: MarcelDekker; 2004.

[13] L. Sciavicco and B. Siciliano, Modeling and Control of Robot Manipulators, 2nd ed. London, U.K.: Springer-Verlag, 2000. 56(5):1213-1219, May 2011. I, III-B, 2, 3

[14] W. Perruquetti and T. Floquet. Homogeneous finite time observer for nonlinear systems with linearizable error dynamics. In Proceedings of the 46th IEEE Conference On Decision and Control, pages 390-395, New Orleans, USA, 2007.

[15] W. Perruquetti, T. Floquet, and E. Moulay. Finite-time observers: Application to secure communication. Automatic Control, IEEE Transactions on, 53(1):356- 360, 2008. I, III-C, III-C3, 8

[16] M. Fliess, C. Join, and H. Sira-Ramirez. Non-linear estimation is easy. International Journal of Modelling, Identification and Control, 4(1):1227, 2008. III-A

[17] M. Mboup, C. Join, and M. Fliess. A revised look at numerical differentiation with an application to nonlinear feedback control. In Control Automation, 2007. MED '07. Mediterranean Conference on Control and automation, pages 1-6, June 2007. I, III-A, 1

[18] K. J. Åström, B. Wittenmark, Adaptive control, Addison-Wesley Longman Publishing Co., Inc. Boston, MA, USA, 1994.

[19] Tira-Thompson E. Digital servo calibration and modeling[J]. Robotics Institute, Pittsburgh, PA, Tech. Rep. CMU-RI-TR-09-41, 2009. 